\title{
Surgimiento y evolución de la ergonomía como disciplina: reflexiones sobre la escuela de los factores humanos y la escuela de la ergonomía de la actividad
}

\author{
Emergence and evolution of ergonomics as a discipline: reflections on the school \\ of human factors and the school of ergonomics of the activity
}

\author{
Surgimento e evolução da ergonomia como disciplina: reflexões sobre a escola \\ dos fatores humanos e a escola de ergonomia da atividade
}

Yaniel Torres ${ }^{1}$, Yordán Rodríguez²

1 Máster en Ingeniería de Riesgos de Seguridad y Salud del Trabajo, máster en Gestión de los Recursos Humanos. École de Technologie Supérieure de Montreal, Canadá. yaniel.torres-medina.1@ens.etsmtl.ca. ORCID: https://orcid.org/0000-0002-9825-9437

2 Doctor en Ciencias Técnicas con énfasis en Ergonomía, máster en Prevención de Riesgos Laborales, máster en Gestión de los Recursos Humanos. Universidad de Antioquia, Medellín, Colombia. yordan.rodriguez@udea.edu.co. oRcid: https://orcid.org/00000002-0079-4336

Recibido: 21/08/2020. Aprobado: 20/03/2021. Publicado: 30/04/2021

Torres Y,RodríguezY. Surgimiento y evolución de la ergonomía como disciplina: reflexiones sobre la escuela de los factores humanos y la escuela de la ergonomía de la actividad. Rev. Fac. Nac. Salud Pública. 2021;39(2):e342868. Dor: https://doi.org/10.17533/ udea.rfnsp.e342868

\section{Resumen}

La ergonomía es considerada, en la actualidad, una disciplina científica consolidada, que se expande continuamente a nivel global. Este escenario actual es el resultado de diferentes visiones que han permeado la evolución de la ergonomía. En este artículo se hace un recorrido histórico de la ergonomía como disciplina, tomando en cuenta la escuela de los factores humanos y la escuela de la ergonomía de la actividad. Se presentan los orígenes de estas escuelas, sus paradigmas subyacentes y se realiza una comparación entre ellas. Las reflexiones presentadas en el artículo en torno a la ergonomía parten de la idea que, desde las diferencias y la diversidad, se erige el desarrollo. Los autores de este artículo son partidarios de abordar la ergonomía como una única disciplina, reconociendo la convergencia y la complementariedad entre las dos escuelas. Más allá de las diferencias existentes, la práctica de la ergonomía debe enfocarse en el diseño de los sistemas de trabajo, tomando como eje central al ser humano. Se espera que estas reflexiones permitan a los profesionales de la ergonomía y de otras diciplinas afines ganar mayor comprensión de cómo abordar la actividad humana para transformarla positivamente. -Palabras clave: ergonomía, factores humanos, estudio del trabajo, interacción hombre-máquina, condiciones de trabajo. 


\begin{abstract}
Ergonomics is now considered a consolidated scientific discipline that is continually expanding globally. This current scenario is the result of different visions that have permeated the evolution of ergonomics. This article presents a historical overview of ergonomics as a discipline considering human factors and the activity-oriented ergonomics schools. The origins of these two schools of thought on ergonomics and their underlying paradigms are presented, and a comparison between them is made. The reflections presented in the article on ergonomics are based on the idea that progress is built on differences and diversity. The authors of this article support
\end{abstract}

the idea of approaching ergonomics as a single discipline, recognizing the convergence and complementarity between the two schools. Beyond the existing differences, ergonomics' practice should be focused on the design of human-centered work systems. It is hoped that the reflections made in this article will enable professionals in ergonomics and other related disciplines to understand how to approach human at work to transform working conditions positively.

-Keywords: ergonomics, human factors, work study, human-machine interaction, working conditions.

\section{Resumo}

A ergonomia é considerada, na atualidade, uma disciplina científica consolidada, que se expande continuamente a nível global. Este cenário atual es el resultado de diferentes visões que han permeado la evolución de la ergonomía. Neste artigo se tem uma recorrido histórico da ergonomia como disciplina, tomando na cuenta a escola dos fatores humanos e a escola da ergonomia da atividade. Se presentan los orígenes de estas escuelas, sus paradigmas subyacentes y se una realiza comparación entre ellas. Las reflexiones presentadas en el artículo en torno a la ergonomía parten de la idea that, from las diferencias y la diversidad, se erige el desarrollo. Los autores de este artículo son partidarios de abordar la ergonomía como una única disciplina, reconociendo la convergencia y la complementariedad entre las dos escuelas. Más allá de las diferencias existentes, la práctica de la ergonomía debe enfocarse en el diseño de los sistemas de trabajo, tomando como eje central al ser humano. Se espera que estas reflexiones permitan a los profesionales de la ergonomía y de otras diciplinas afines ganar mayor comprensión de cómo abordar la actividad humana para transformarla positivamente.

------ Palabras clave: ergonomía, fatores humanos, estudio del trabajo, interacción hombre-máquina, condiciones de trabajo

\section{Introducción}

La asociación entre el trabajo y diversos tipos de enfermedades ocupacionales, incluidas las lesiones musculoesqueléticas, fue documentada por primera vez hace ya al menos tres siglos. Bernardino Ramazzini (16331714), un médico italiano, observó durante su práctica médica la prevalencia de determinados tipos de dolencias que estaban asociados a trabajos específicos realizados por la población tratada. Ramazzini describe en detalle sus observaciones en la publicación de 1700, De morbis artificum diatriba (Discurso sobre las enfermedades de los trabajadores). Este libro es considerado el principal antecedente histórico de la medicina del trabajo moderna y a Ramazzini se le tiene como el padre de la medicina del trabajo.

En su obra, Ramazzini destacaba: "Ahora quiero referirme a los trabajadores en los que ciertas afecciones mórbidas surgen gradualmente de alguna postura particular de las extremidades o de movimientos antinaturales del cuerpo que se requieren mientras trabajan" [1]." En esta frase, Ramazzini relaciona los factores laborales y la aparición de desórdenes musculoesqueléticos (DME), temática que desde hace varias décadas es de gran interés para la disciplina y la profesión de la ergonomía, al igual que un importante problema de salud pública.

Sin embargo, mirando en retrospectiva, tuvieron que pasar muchos años desde la publicación del libro de Ramazzini (año 1700), para que se hiciera referencia por primera vez al término "ergonomía", lo cual ocurrió en 1857, atribuido a Wojciech Jastrzebowski (1799-1882) [2]. El término fue postulado por este científico naturalista polaco como una derivación del griego ergon (trabajo) y nomos (leyes), identificando de esta manera la ergonomía como la ciencia del trabajo.

* Traducido por los autores 
Curiosamente, no es hasta el año 1949, casi un siglo después de su primera aparición, que el término "ergonomía" se retoma y su definición se precisa a la luz de la modernidad. Esta vez fue el psicólogo inglés Hywel Murrell (1908-1984) quien reintroduce la palabra "ergonomía" durante una reunión en la sede de la Marina Real Británica (British Admiralty), donde trabajaba como científico. Esta reunión sentó las bases para la fundación, en el mismo año 1949, de la primera sociedad de ergonomía en el mundo, la Ergonomics Research Society, del Reino Unido [3].

Murrell define la ergonomía como el estudio científico de la relación entre el hombre y su ambiente de trabajo. La palabra "ambiente" se emplea para indicar no solo el medio físico, sino también las herramientas y los materiales, así como los métodos y la organización del trabajo en los ámbitos tanto individual como grupal [4]. De esta manera, la ergonomía emerge como disciplina científica a finales de la década de los cuarenta, como resultado del creciente aumento de la complejidad de los sistemas tecnológicos. Esto era más evidente en el sector militar, en el cual las demandas físicas y cognitivas del operador humano eran elevadas. El sector militar comprendió que era necesario tener en cuenta las características de las personas en sus diseños, para así aprovechar el potencial del equipamiento bélico. A medida que los logros tecnológicos de la Segunda Guerra Mundial se trasladaron a las aplicaciones civiles, se encontraron los mismos problemas asociados a ciertas incompatibilidades entre las personas y el equipamiento, con un impacto negativo en el desempeño y un mayor riesgo de error humano [5]. Rápidamente, la importancia de la ergonomía fue acogida en diversas regiones del planeta. En 1957, se funda, en Estados Unidos, la Sociedad de Ergonomía y Factores Humanos (Human Factors and Ergonomics Society, HFES). En el año 1961, se crea la Asociación Internacional de Ergonomía (Internacional Ergonomics Association, IEA), y en 1963, se funda la Sociedad de Ergonomía en Lengua Francesa (Société d'Ergonomie de Langue Française, SELF). En la actualidad, más de 50 sociedades de ergonomía de diversas regiones del mundo forman parte integral de la IEA.

La ergonomía, durante su evolución, se ha nutrido de varias disciplinas científicas y ha contribuido a otras, como la salud pública. Por ejemplo, la aplicación de los principios de ergonomía en el diseño de los sistemas de trabajo ha ayudado a disminuir la fatiga, el malestar, la carga de trabajo, las lesiones y los trastornos crónicos que pueden padecer la población trabajadora.

Un tema de especial interés para la ergonomía, y que es considerado también un asunto de salud pública, son los DME de origen laboral (ej. síndrome del túnel del carpo, tendinitis, lumbalgias, bursitis, etc.). Estos afectan a trabajadores tanto de países desarrollados como subde- sarrollados, lo que genera un elevado impacto socioeconómico a nivel global. Instituciones e investigadores de varias partes del mundo reconocen que la aplicación de los principios de la ergonomía es una de las maneras más efectivas de prevenir y mitigar esta pandemia ocupacional que constituyen los DME $[6,7]$.

La ergonomía, desde su enfoque de sistemas, no solo contribuye a resolver problemas de salud pública de origen laboral, sino también aquellos relacionados con la vida diaria de las personas, como el mejoramiento de los servicios de salud (ej. errores de medicación, calidad asistencial, diseño de instalaciones y equipos médicos, diseño de procedimientos seguros, entre otros). En este sentido, la Organización Mundial de la Salud reconoce a la ergonomía como una de las estrategias y disciplinas claves, en su propuesta de estrategia global (2021-2030) para mejorar la seguridad del paciente [8].

Otra temática de interés para la ergonomía son los horarios de trabajo. El trabajo por turnos puede ocasionar diversos problemas de salud en la población trabajadora: trastornos del sueño, trastornos digestivos, enfermedades cardiovasculares y desórdenes metabólicos, como la diabetes tipo 2 [9]. En estos casos, la ergonomía busca optimizar la estructura de los horarios de trabajo, de tal manera que se minimicen los problemas de salud.

Otro elemento relevante de la ergonomía es su naturaleza inclusiva, ya que toma en cuenta la variabilidad humana en el diseño de los sistemas. Por ejemplo, algunas temáticas contemporáneas de la ergonomía incluyen el diseño para personas con discapacidades tanto físicas como mentales, así como para la población de avanzada edad. De esta manera, la ergonomía contribuye a mejorar la salud, la seguridad y el bienestar de las personas, impactando en la salud pública.

Durante el desarrollo histórico de la ergonomía como disciplina, surgieron dos escuelas de pensamiento con características distintivas: la escuela de los factores humanos y la escuela de la ergonomía de la actividad. Esta última, también conocida como "escuela francesa", ha sido poco difundida, en especial en el continente americano. Por esta razón, en ocasiones se desconocen los principales elementos que la conforman, así como su enfoque en cuanto al abordaje del ser humano en el trabajo.

En este artículo se hace un recorrido histórico de la ergonomía como disciplina, considerando la escuela de los factores humanos y la escuela de la ergonomía de la actividad. Se presentan los orígenes de estas escuelas, sus paradigmas subyacentes y se hace una comparación entre ellas. El artículo no pretende ser una descripción exhaustiva de los dos enfoques, sino servir de referencia a los profesionales interesados en la ergonomía, sobre las características de estas dos escuelas de pensamiento. 
Los autores de este artículo parten del supuesto de que estas dos escuelas son complementarias, y ambas tienen mucho que aportar a la práctica de la ergonomía desde sus enfoques y visiones respectivas.

\section{Ergonomía de los factores humanos: orígenes y evolución}

La escuela de la ergonomía de los factores humanos se desarrolló fundamentalmente en los países occidentales anglosajones, como Estados Unidos, el Reino Unido y otros países europeos $[5,10,11]$.

Desde el punto de vista evolutivo, el surgimiento de esta escuela de pensamiento de la ergonomía puede verse como una respuesta a las limitaciones de la organización científica del trabajo (Scientific Management) que se produjo a finales del siglo XIX y principios del Xx, y tenía como principal objetivo mejorar la eficiencia del trabajador.

Frederick Winslow Taylor (1856-1915) es considerado el padre de la organización científica del trabajo. Para este ingeniero mecánico, siempre se debía buscar una manera más eficiente de realizar una tarea, con el fin de economizar tiempo y aumentar la productividad [12]. Ciertamente, las ideas propuestas por Taylor tuvieron gran impacto en la racionalización de la producción y en el desarrollo de las técnicas de producción en masa. Gracias a sus aportes, así como a los avances introducidos por Henry Ford en la cadena de montaje, se inició la era industrial, caracterizada por una elevada productividad y la fabricación de bienes de consumo cada vez más accesibles.

Sin embargo, las ideas de Taylor también crearon resentimiento y oposición entre los trabajadores de las industrias modernas de producción, ya que se enfocaban en optimizar el trabajo para mejorar la eficiencia productiva, sin tomar en cuenta los efectos negativos sobre el individuo. Taylor, en particular, ha sido reprochado por su obra The principles of scientific management (Los principios de la organización científica del trabajo), ${ }^{*}$ como consecuencia de algunos pasajes con connotaciones políticas capitalistas y críticas a la clase trabajadora [13].

No obstante, es importante reconocer que Taylor llevó a cabo uno de los primeros intentos en la aplicación de la ciencia al estudio de los procesos de producción y del trabajo humano en particular. Más bien, Taylor debe ser criticado a la luz de los conocimientos existentes en el momento histórico que le tocó vivir hace más de 100 años. Muchas de las disciplinas sobre las cuales se basan las críticas a Taylor, como la biomecánica, la antropometría, la fisiología del trabajo o la psicología del trabajo no existían en su tiempo o apenas estaban en sus orígenes. Al ser ingeniero mecánico de formación, su comprensión del trabajo humano estaba indudablemente sesgada por un enfoque mecanicista.

Los esposos Frank Bunker Gilbreth (1868-1924) y Lillian Evelyn Gilbreth (1878-1972) expandieron las ideas iniciadas por Taylor y desarrollan las técnicas clásicas de estudio de tiempos y movimientos, que constituyen la base de la medición del trabajo [14]. Los conceptos manejados por los esposos Gilbreth se estiman mucho más cercanos a la ergonomía moderna, pues no solo tenían en cuenta la eficiencia del trabajo, sino también el bienestar de las personas [15].

Desde un punto de vista histórico, la ergonomía de los factores humanos está vinculada a la organización científica del trabajo, y pudiera asumirse como la evolución de un enfoque fundamentalmente centrado en la eficiencia del trabajo a un enfoque centrado en el humano. De esta manera, se trasciende la visión mecanicista del trabajo, para tomar en cuenta los límites tanto físicos como cognitivos de los humanos.

Por ejemplo, Hywel Murrell, el padre fundador de la ergonomía moderna, desarrolló su interés por la disciplina de la ergonomía como resultado de su trabajo en el grupo de investigación de operaciones del ejército británico durante la Segunda Guerra Mundial. Murrell empleaba un enfoque basado en estudios de tiempo y movimientos (motion and time study) para analizar el comportamiento humano en un ambiente de trabajo [16]. Posteriormente, en 1947, Murrell fue nombrado jefe de la Naval Motion Study Unit (Unidad de Estudio de Movimiento) de la Marina Británica. De esta manera, pudiera suponerse que Murrell llegó a comprender los límites de la organización científica del trabajo, y que su formación como psicólogo le brindó la visión necesaria para hacer evolucionar su enfoque sobre el análisis del trabajo.

El vínculo entre la ergonomía de los factores humanos y la organización científica del trabajo se manifiesta en los programas de estudio modernos de especialidades como la Ingeniería Industrial y de Sistemas [10]. En ellos, si bien la ergonomía se centra en el humano y la optimización del sistema de trabajo, elementos como la calidad, la productividad, el costo de producción y la eficiencia son de gran relevancia.

Es importante destacar que la ergonomía de los factores humanos es considerada el enfoque prevaleciente en la mayor parte de las economías industrializadas, así como en la mayoría de los países en los cuales esta disciplina tiene presencia. Dada la influencia comercial de Estados Unidos en Latinoamérica, era de esperarse que la corriente de la ergonomía de los factores humanos se expandiera al sur de sus fronteras.

* Traducido por los autores. 
Se plantea la hipótesis que esta expansión se produce en especial a través de programas de formación de Ingeniería Industrial, que tienen sus orígenes en Estados Unidos [17], pero también mediante modelos de gestión de compañías multinacionales que operan en territorio latinoamericano y que toman en cuenta el estudio del trabajo, los procesos y la ergonomía.

\section{Ergonomía de la actividad o escuela francófona}

La escuela de la ergonomía de la actividad se originó en Francia y ha sido adoptada mayoritariamente en países de habla francesa: Bélgica, Suiza, Canadá, entre otros [18].

La ergonomía de la actividad ha desarrollado un cuerpo de conocimientos propios, llegando a contar incluso con una sociedad (SELF), y aunque pudiera pensarse que la diferencia radica fundamentalmente en la lengua en la cual se trabaja, en realidad la diferencia con la escuela de los factores humanos parece ser más profunda.

Desde el punto de vista histórico, en 1955, André Ombredane y Jean-Maire Faverge publican L'analyse du travail (El análisis del trabajo), obra que se constituye en el antecedente del posterior desarrollo de la ergonomía de la actividad [19].

En la década de los setenta, la ergonomía desarrollada en Francia incorpora fundamentos de la psicología del trabajo soviética, provenientes de diversos estudios realizados en la antigua Unión Soviética, que tuvieron eco en los países nórdicos, así como en la propia Francia, donde hubo un marcado interés por el desarrollo de estas ideas [20].

Según González [21], la ergonomía de la actividad tiene como categoría central "el trabajo", considerándose su principal objeto teórico. Esta escuela respalda la tesis que trabajar equivale a gestionar una tensión resultante de un doble requerimiento: por un lado, un requerimiento de efectividad, que se refiere a las expectativas de la empresa hacia el trabajador y se expresa mediante la tarea asignada; y, por otro, un requerimiento de conservación de la salud, que alude a las expectativas de la persona, y se manifiesta a través de su involucramiento en la actividad de trabajo. De manera sintética, el trabajador debe mantener un equilibrio entre las exigencias de la tarea y la conservación de su estado de salud. Esta teoría es conocida como "teoría de la doble regulación" y es clave para entender los basamentos teóricos de la escuela de la ergonomía de la actividad [22].

El centro del enfoque de esta escuela lo constituye el concepto de actividad de trabajo, que enfatiza en la diferencia entre el trabajo prescrito y el trabajo real [23]. El trabajo prescrito está asociado a lo que el trabajador debe ejecutar siguiendo un grupo de instrucciones o método de trabajo preestablecido en un contexto dado y con herramientas predefinidas. El trabajo real, por otro lado, representa el cómo el trabajador ejecuta en la práctica estas instrucciones que le son transmitidas a través de los procedimientos y normas de trabajo.

La ergonomía de la actividad considera, ante todo, al trabajador como un agente activo, capaz de poner en práctica una gran variedad de estrategias para cumplir con la demanda impuesta por la tarea, y de esta manera ampliar su margen de maniobra. El concepto de margen de maniobra es, en esencia, la diferencia entre la demanda impuesta al trabajador por la tarea (trabajo prescrito) y su capacidad de hacerle frente a esta demanda (trabajo real), y es otro de los conceptos claves manejados por la ergonomía de la actividad [24].

Esta escuela se interesa particularmente en las estrategias desarrolladas por los trabajadores para aumentar su capacidad de hacerle frente a las demandas impuestas por el trabajo (margen de maniobra). Estas estrategias no son, en ocasiones, fácilmente visibles y pueden escapar a la observación, por lo que se han desarrollado técnicas para extraer este conocimiento tácito que el trabajador lleva consigo.

Durante este proceso, la ergonomía de la actividad brinda un rol preponderante a la figura del trabajador. En este sentido, el concepto de ergonomía participati$v a$, que se ha ido desarrollando a través de los años en la ergonomía de los factores humanos, ya existía en la naturaleza misma de la ergonomía de la actividad [25].

Por otro lado, la ergonomía de la actividad tiene una fuerte influencia de las ciencias humanas o sociales. Según González [21], las principales técnicas empleadas en la ergonomía francófona se pueden dividir en: observaciones de la actividad, entrevistas a los trabajadores y simulaciones de situaciones de trabajo.

Para ganar en comprensión sobre los elementos subyacentes a la escuela de la ergonomía de la actividad, se debe señalar que varios de los pioneros de esta corriente de pensamiento fueron reconocidos militantes de izquierda de tendencia marxista [26]. Tal es el caso de Antoine Laville (1934-2002) y Alain Wisner (1923-2004), los cuales desarrollaron sus teorías en el laboratorio de fisiología del trabajo del Conservatoire Nationaldes Arts et Métiers (CNAM) en París, durante la década de los sesenta [27]. Con frecuencia, sus trabajos se desarrollaron en colaboración y según la demanda de grandes sindicatos franceses, como, por ejemplo, el sindicato francés de trabajadores ferroviarios [26].

La ergonomía de la actividad ha tratado sistemáticamente algunos temas que han quedado fuera del campo de análisis de la ergonomía de los factores humanos. Un ejemplo lo constituye el enfoque de género en el análisis de las situaciones de trabajo, propuesto por esta escuela e impulsado por investigadores de Quebec [28,29]. Se ha mencionado, en la literatura, que es probable que las mujeres experimenten dificultades en sectores de acti- 
vidad que no han alcanzado una cierta masa crítica de mujeres [28]. Por lo tanto, mientras sean una minoría en un sector laboral, pueden encontrar obstáculos. Uno de estos puede ser la inadaptación de herramientas de trabajo que han sido diseñadas para uso de hombres, y que no toman en cuenta las posibles diferencias antropométricas y biomecánicas entre los dos géneros.

Otro tema clave, sobre el que se ha desarrollado todo un campo de investigación, es el relacionado con la intervención en ergonomía. En este caso, los investigadores se han interesado ampliamente por el proceso de intervención ergonómica, el cual va más allá del análisis de la actividad de trabajo en contexto real y comprende la implicación de diversos actores del sistema objeto de estudio [30,31]. En este sentido es analizada la sostenibilidad en el tiempo de las acciones de intervención, con el objetivo de sensibilizar a los diversos actores en la organización. El centro lo constituye la construcción social de la intervención ergonómica. Por ejemplo, Coutarel et al. [32] proponen pautas para evaluar intervenciones enfocadas en la prevención de DME relacionados con el trabajo desde el punto de vista de la transformación organizacional.

Algunos artículos de la escuela de la ergonomía de la actividad han llegado incluso a plantear debates entre fenómenos como la globalización y las condiciones de trabajo [33]. Este tipo de análisis de relaciones jerárquicas y de estructuras asociadas al funcionamiento sociopolítico y económico está más alineado con las ciencias sociales que con las ciencias técnicas. Temas como el sufrimiento en el trabajo, la representatividad de género, la representación social de los actores, la globalización, las relaciones jerárquicas, son frecuentes en esta escuela de la ergonomía surgida en Francia. Un recorrido por algunas revistas marcadas por la ergonomía de la actividad, como PISTES, Le travail humain o Activités, permite constatar una representación importante de debates de tipo macroconceptuales y epistemológicos sobre el rol del trabajo, el trabajador y el sistema social en general.

La ergonomía de la actividad en ocasiones cohabita con la escuela de los factores humanos, como es el caso de Canadá, particularmente en la provincia de Quebec. Allí existen tanto programas de enseñanza basados en los enfoques de la ergonomía de los factores humanos como de la ergonomía de la actividad. Esto último, por la influencia que la escuela francesa ha tenido en la única región en Norteamérica donde la lengua oficial es el francés y en la segunda metrópolis francófona más importante del mundo después de París: la ciudad de Montreal.

Brasil constituye un caso peculiar en América Latina, pues aunque el desarrollo de la ergonomía ha tenido fuerte influencia del campo de las ingenierías [34], también se ha desarrollado una vertiente de la escuela de la ergonomía de la actividad. Los antecedentes de la influencia de esta escuela de la ergonomía en Brasil se encuentran en la visita que hiciese uno de los grandes exponentes de la ergonomía de la actividad en Francia y en el mundo: Alain Wisner, del CNAM.

Animados por el profesor Wisner, varios investigadores brasileños fueron a Francia para asistir a cursos de posgrado en ergonomía en el CNAM, en París. A su regreso, estos profesores fueron responsables del desarrollo de varios programas de investigación en universidades brasileñas [35].

Un proceso similar, aunque a menor escala, se dio igualmente en Argentina, con la visita de un grupo de destacados ergónomos franceses, incluyendo los dos antes mencionados. Asimismo, varios profesionales de la ergonomía en Argentina se formaron en Francia, como parte de la colaboración inicialmente establecida [36].

También algunos profesionales de Venezuela (Universidad de Carabobo) y de Chile (Universidad de Atacama) se han formado en Quebec, en el marco de proyectos de colaboración internacional [37].

\section{Síntesis comparativa entre las dos es- cuelas de la ergonomía}

La principal diferencia entre las dos escuelas no viene dada por la definición misma de lo que constituye la disciplina de la ergonomía ni su objeto de estudio, como se muestra en la Tabla 1. En esta tabla se resaltan los términos relevantes que se repiten en las definiciones brindadas por las distintas asociaciones de ergonomía. Se aprecia que la esencia de estas definiciones es muy similar, lo que podría hacernos cuestionar sobre cuán diferente puede ser la ergonomía de los factores humanos con respecto a la ergonomía de la actividad. La diferencia esencial entre las dos escuelas de la ergonomía viene dada, al parecer, por la forma de abordar la problemática de la interacción del humano con su entorno de trabajo.

De manera general, la escuela de la ergonomía de la actividad pone gran énfasis en el aspecto descriptivo del análisis de las situaciones de trabajo, empleando herramientas de tipo cualitativas en las cuales el trabajador tiene gran preponderancia. Tal es el caso de las observaciones antropológicas o etnográficas, que ayudan a explicitar conocimiento tácito y pueden ser empleadas para transmitir conocimientos entre trabajadores experimentados y novatos mediante formaciones adaptadas.

Hasta el presente, la ergonomía de la actividad se ha insertado principalmente en países desarrollados que cuentan con gran madurez en los procesos de análisis de situaciones de trabajo. Es de esperarse que el empleo de la ergonomía de la actividad pueda tener desventajas si no es usado en el marco de una intervención más global que tome en cuenta diversos aspectos, como la cuantificación del riesgo y elementos o variables asociadas a los procesos productivos o de servicios. En un sistema de producción 
Tabla 1. Definiciones de ergonomía brindadas por tres de las más importantes asociaciones a escala global.

\begin{tabular}{|c|c|}
\hline Sociedad & Definición de ergonomía* \\
\hline $\begin{array}{l}\text { Asociación Internacional } \\
\text { de Ergonomía (IEA) }\end{array}$ & $\begin{array}{l}\text { "La ergonomía (o factores humanos) es la disciplina científica que se ocupa de la comprensión } \\
\text { de las interacciones entre los humanos y otros elementos del sistema, y a la vez la profesión } \\
\text { que aplica teorías, principios, datos y métodos al diseño con el objetivo de optimizar el } \\
\text { bienestar humano y el desempeño del sistema global. Los ergónomos contribuyen al diseño y } \\
\text { la evaluación de tareas, trabajos, productos, ambientes y sistemas con el objetivo de hacerlos } \\
\text { compatibles a las necesidades, habilidades y limitaciones de las personas" [38] }\end{array}$ \\
\hline $\begin{array}{l}\text { Sociedad de Ergonomía y } \\
\text { Factores Humanos (HFES) }\end{array}$ & $\begin{array}{l}\text { "La ergonomía y los factores humanos emplean conocimiento sobre las habilidades humanas y } \\
\text { sus limitaciones en el diseño de sistemas, organizaciones, trabajos, maquinarias, herramientas y } \\
\text { productos encaminados a un uso humano de manera segura, eficiente y confortable" [11]. }\end{array}$ \\
\hline $\begin{array}{l}\text { Sociedad de Ergonomía } \\
\text { en Lengua Francesa (SELF) }\end{array}$ & $\begin{array}{l}\text { "La puesta en práctica de conocimientos científicos relativos al ser humano y necesarios para } \\
\text { el diseño de herramientas, de maquinarias y de dispositivos que podrán ser utilizados por la } \\
\text { mayoría de los individuos con un máximo de confort, de seguridad y de eficacidad" [18]. }\end{array}$ \\
\hline
\end{tabular}

* La traducción de las definiciones y los textos resaltados fueron realizados por los autores.

marcadamente capitalista, en el cual la competitividad de las compañías es un elemento clave, la productividad, la calidad y el costo de producción seguirán siendo aspectos relevantes para los gestores de las organizaciones y deben ser, por ende, considerados en la práctica de la ergonomía.

Por otro lado, la escuela de los factores humanos está estrechamente vinculada al desarrollo del estudio científico del trabajo y toma un antecedente fuerte en las ciencias técnicas de tipo ingenieril, mientras que la escuela de la ergonomía de la actividad encuentra un antecedente importante en las ciencias sociales y humanas. La escuela de los factores humanos favorece el uso de técnicas cuantitativas, mientras que la escuela de la ergonomía de la actividad favorece las técnicas cualitativas.

Pudiera decirse que el enfoque de la escuela de los factores humanos está más armonizado con el lenguaje empleado por los ingenieros y los gestores en las diversas organizaciones en términos de calidad, productividad y otros aspectos del desempeño del sistema de producción o servicio. Se le ha criticado, en ocasiones, por ser un enfoque de tipo tecnocrático, con limitado énfasis en el trabajador, pero hay evidencia de que ha evolucionado hacia modelos más participativos $[39,40]$.
En el caso de la escuela de la ergonomía de la actividad, algunas limitaciones podrían estar asociadas a la preponderancia brindada al trabajador durante el análisis de las situaciones de trabajo, el uso de un vocabulario y conceptos proveniente de ciencias humanas en un contexto organizacional, y el peso importante brindado a elementos de tipo descriptivo en relación con la cuantificación.

En la Tabla 2 se hace un resumen de las principales características de cada enfoque, mostrando de manera sintética algunas diferencias entre estas dos escuelas.

Si tomamos como punto de partida de la ergonomía moderna a Murrell, se debe precisar que, en su obra de 1965, el autor plantea en referencia a la disciplina: "Periféricas a la ergonomía, pero no consideradas en la actualidad como parte de este campo, están las relaciones del trabajador con sus colegas de trabajo, supervisores, gestores y su familia. Estos temas son tratados generalmente como parte de las ciencias sociales, pero no pueden ser ignorados ya que pudieran desempeñar un rol importante en la solución de algunos problemas de ergonomía" [4].

Dada la marcada presencia de las ciencias sociales en la ergonomía de la actividad, cabe preguntarse si estos temas asociados a las relaciones humanas en el trabajo han dejado de ser periféricos y se han convertido

Tabla 2. Resumen de las principales características de los dos enfoques.

\begin{tabular}{lll}
\hline \multicolumn{1}{c}{ Característica } & \multicolumn{1}{c}{ Ergonomía de los factores humanos } & \multicolumn{1}{c}{ Ergonomía de la actividad } \\
\hline Antecedente histórico & Estudio científico del trabajo & Psicología del trabajo soviética \\
Disciplinas de influencia & Ciencias técnicas & Ciencias humanas o sociales \\
Tipo de técnicas preponderantes & Mixto, con énfasis en técnicas cuantitativas & Mixto, con énfasis en técnicas cualitativas \\
Rol del trabajador & Participación directa o indirecta según el caso & $\begin{array}{l}\text { Enfoque antropológico, con participación } \\
\text { directa }\end{array}$ \\
\hline
\end{tabular}


en parte integral del objeto de la ergonomía. No obstante, entre las definiciones más actuales de la ergonomía como disciplina científica, incluyendo la de la SELF, no se mencionan temas vinculados a las relaciones humanas en el trabajo (jerarquía, género o aspectos culturales, entre otros). Sin embargo, no tomar en cuenta estos elementos y emplear un enfoque centrado únicamente en la escuela de los factores humanos podría limitar el poder de actuar de los profesionales de la ergonomía en organizaciones que responden a estructuras sociales que se deben considerar.

\section{Conclusión}

A partir de la idea de que desde las diferencias y la diversidad se erige el desarrollo, en este artículo se hacen reflexiones acerca del surgimiento y la evolución de la ergonomía como disciplina, desde el enfoque de la escuela de los factores humanos y de la escuela de la ergonomía de la actividad.

Los autores somos partidarios de abordar la ergonomía como una única disciplina, reconociendo la necesidad de convergencia y de complementariedad entre las dos escuelas.

Más allá de las diferencias existentes, la práctica de la ergonomía debe estar enfocada en el diseño del sistema de trabajo centrado en el ser humano, para, de esta manera, contribuir al bienestar, la salud y la seguridad de la población trabajadora.

Se espera que las reflexiones presentadas en este artículo permitan a los profesionales de la ergonomía y de otras diciplinas afines ganar mayor comprensión de cómo abordar la actividad humana para transformarla positivamente. Al fin y al cabo, una de las obras claves de la ergonomía de la actividad se titula Comprendre le travail pour le transformer que significa en español: Comprender el trabajo para transformarlo [41]. Una comprensión profunda del trabajo que no genere transformación palpable para el trabajador no respondería al carácter dinámico de la disciplina de la ergonomía.

\section{Declaración de fuente de financiación}

Este trabajo no contó con ninguna fuente de financiación.

\section{Declaración de conflictos de intereses}

Los autores declaran no tener conflicto de intereses.

\section{Declaración de responsabilidad}

Los puntos de vista expresados son de los autores y no de las instituciones a las que pertenecen.

\section{Declaración de contribución por autores}

Los dos autores cumplen los cuatro criterios de autoría según ICMJE.

\section{Referencias}

1. Franco G, Franco F. Bernardino Ramazzini: The father of occupational medicine. Am J Public Health. 2001;91(9):1382. Dor: https://doi.org/10.2105/AJPH.91.9.1382

2. Karwowski W. Ergonomics and human factors: The paradigms for science, engineering, design, technology and management of human-compatible systems. Ergonomics. 2005;48(5):436-63. DOI: https://doi.org/10.1080/00140130400029167

3. Chartered Institute of Ergonomics \& Human Factors. Who was Hywel Murrell? [internet]; 2019 [citado 2020 jul. 4]. Disponible en: http://bit.ly/Hywel_Murrell

4. Murrell K. Ergonomics. Man in his working environment. London, U.K.: Chapman and Hall Ltd.; 1965.

5. Chartered Institute of Ergonomics \& Human Factors. A brief history [internet]; 2018 [citado 2020 abr. 23]. Disponible en: http:// bit.ly/CIEHF_A_Brief_History

6. Dennerlein JT. Ergonomics and musculoskeletal issues. In: Quah SR, editor. International encyclopedia of public health (2. ${ }^{a}$ ed.). Oxford: Academic Press; 2017. pp. 577-84.

7. Torres Y, Rodríguez Y, Viña S. Preventing work-related musculoskeletal disorders in Cuba, an industrially developing country. Work: A Journal of Prevention, Assessment and Rehabilitation. 2011;38(3):301-6. DoI: https://doi.org/10.3233/WOR-2011-1133

8. World Health Organization (wHO). Global patient safety action plan 2021-2030: Towards zero patient harm in health care. Geneva: wно; 2020.

9. Moreno CRC, Marqueze EC, Sargent C, et al. Working time society consensus statements: Evidence-based effects of shift work on physical and mental health. Ind Health. 2019;57(2):139-57. Dor: https://doi.org/10.2486/indhealth.SW-1

10. Institute of Industrial System Engineers (IISE). Body of knowledge [internet]; 2018 [citado 2020 abr. 20]. Disponible en: http://www. iise.org/details.aspx?id=43631

11. Human Factors and Ergonomics Society (HFEs). HFEs History [internet]; 2018 [citado 2020 may. 20]. Disponible en: https://www. hfes.org/About-HFES/HFES-History

12. Kanigel R. The one best way: Frederick Winslow Taylor and the enigma of efficiency. Cambridge, MA: MIT Press; 2005.

13. Taylor FW. The principles of scientific management. New York, NY: Harper \& Brothers; 1911.

14. Gilbreth FB. Primer of scientific management. New York, NY: Van Nostrand; 1914.

15. Nadworny MJ. Frederick Taylor and Frank Gilbreth: Competition in scientific management. The Business History Review. 1957;31(1):23-34. DoI: https://doi.org/10.2307/3111727 
16. Fletcher C. Hywell Murrell: One of the founders of ergonomics and what we now call Human Factors. British Psichological Society Files [internet]. 2016 [citado 2020 jun. 14]. Disponible en: http://bit.ly/Hywell_Murrell_BPS

17. Blúa L. La inserción de la ingeniería industrial en América Latina Prezi [internet]; 2014 [citado 2020 abr. 27]. Disponible en: http:// bit.ly/Ingenieria_Industrial_AL

18. Société d'Ergonomie de Langue Française. Mission [internet]. 2018 [citado 2020 mar. 26]. Disponible en: https://ergonomie-self. org/la-self/mission/

19. Ombredane A, Faverge J. L'analyse du travail. Paris: Presses Universitaires de France; 1955.

20. Daniellou F, Rabardel P. Activity-oriented approaches to ergonomics: Some traditions and communities. Theor Issues Ergon Sci. 2005;6(5):353-7. DoI: https://doi. org/10.1080/14639220500078351

21. González RE. Aportes de la ergonomía a la comprensión y transformación de las condiciones de trabajo: una aproximación económica, humanista, política y social del estudio del trabajo. Salud de los Trabajadores. 2002;10(1-2):47-62.

22. Veyrac H, Bouillier-Oudot M-H. Les concepts de représentations de la tâche en ergonomie pour la formation professionnelle des enseignants débutants. In: Maubant P, Martineau S, editors. Fondements des pratiques professionnelles des enseignants. Ottawa: Presses de l'Université d'Ottawa; 2011. pp. 219-42.

23. Barbier J-M, Durand M. L'activité : un objet intégrateur pour les sciences sociales? Recherche \& Formation. 2003;42:99-117.

24. Vézina N, editor. La Pratique de l'ergonomie face aux tms: Ouverture à l'interdisciplinarité. Comptes rendus du congrès SELFACE 2001 - Les transformations du travail, enjeux pour l'ergonomie; 2001; Montréal, Canada.

25. Hubault F, Bourgeois F. Disputes sur l'ergonomie de la tâche et de l'activité, ou la finalité de l'ergonomie en question. Activités. 2004;1(1):34-53. Dor: https://doi.org/10.4000/activites.1149

26. Daniellou F. The French-speaking ergonomists' approach to work activity: Cross-influences of field intervention and conceptual models. Theor Issues Ergon Sci. 2005;6(5):409-27. Dor: https://doi. org/10.1080/14639220500078252

27. Dejours C. Alain Wisner, une démarche une référence. Travailler. 2006;15(1):9-10. DoI: https://doi.org/10.3917/trav.015.0009

28. Messing K, Seifert AM, Couture V. Les femmes dans les métiers non-traditionnels : le général, le particulier et l'ergonomie. Travailler. 2006;15(1):131-47. DoI: https://doi.org/10.3917/ trav.015.0131
29. Messing K, Seifert AM. "On est là toutes seules ". Contraintes et stratégies des femmes en contrat à durée déterminée dans l'enseignement aux adultes. Travailler. 2002;1(7):147-66. Dor: https:// doi.org/10.3917/trav.007.0147

30. St-Vincent M, Vézina N, Laberge M, et al. L'intervention ergonomique participative pour prévenir les TMS. Ce qu'en dit la littérature francophone. Montréal: Institut de recherche Robert-Sauvé en santé et en sécurité du travail; 2010.

31. St-Vincent M, Imbeau D, Denis D, et al. Suivi de huit interventions de prévention des TMS initiées par des inspecteurs de la CSST: étude exploratoire. Montréal: Institut de recherche Robert-Sauvé en santé et en sécurité du travail; 2009.

32. Coutarel F, Vézina N, Berthelette $D$, et al. Orientations pour l'évaluation des interventions visant la prévention des troubles musculo-squelettiques liés au travail. PISTES. 2009;11(2). DOI: https://doi. org/10.4000/pistes. 2349

33. Seifert AM, Messing K. Cleaning up after globalization: An ergonomic analysis of work activity of hotel cleaners. Antipode. 2006;38(3):557-78. DoI: https://doi.org/10.1111/j. 0066-4812.2006.00595.x

34. Santana C, Fialho FAP. The History of Ergonomics in Brazil. Proc Hum Factors Ergon Soc Annu Meet. 2000;44(33):6-96-6-9. Dor: https://doi.org/10.1177/154193120004403303

35. Soares M. Ergonomics in Brazil and the Brazilian ergonomics society (Abergo). Boca Raton, FL: CRC Press; 2006.

36. Soares MM. Ergonomics in Latin America: Background, trends and challenges. Applied Ergonomics. 2006;37(4):555-61. DOI: https://doi.org/10.1016/j.apergo.2006.04.014

37. González R, Weill-Fassina A. Évolution des analyses de l'activite en amerique du sud d'apres les communications presentees a la self (1988-2002). XXXVIIIème Congrès de la Société d'Ergonomie de Langue Française. Paris: SELF; 2003.

38. International Ergonomics Association (IEA). Human Factors/Ergonomics (HF/E) [internet]; 2000 [citado 2020 jun. 10]. Disponible en: https://iea.cc/what-is-ergonomics/

39. Brown O. Marketing participatory ergonomics: Current trends and methods to enhance organizational effectiveness. Ergonomics. 1990;33(5):601-4. DOI: https://doi. org $/ 10.1080 / 00140139008927169$

40. Hignett S, Wilson JR, Morris W. Finding ergonomic solutions-participatory approaches. Occup Med (Lond). 2005;55(3):200-7. DOI: https://doi.org/10.1093/occmed/kqi084

41. Guérin F, Laville A, Daniellou F. Comprendre le travail pour le transformer: la pratique de l'ergonomie. Lyon, France: Editions ANACT; 1997. 